\title{
Estrés postraumático, ansiedad, afrontamiento y resiliencia en escolares tras el terremoto de 2017 en México
}

\author{
Post-traumatic stress, anxiety, coping, and resilience \\ in schoolchildren post 2017 earthquake in Mexico
}

\author{
Norma Ivonne González-Arratia López-Fuentes, \\ Martha Adelina Torres Muñoz y Felipe González-Arratia Visoso ${ }^{l}$
}

Citación: González-Arratia L.F., N.I., Torres M., M.A. y González-Arratia V., F. (2021). Estrés postraumático, ansiedad, afrontamiento y resiliencia en escolares tras el terremoto de 2017 en México. Psicología y Salud, 31(1), 15-24. https://doi.org/10.25009/pys.v31i1.2672.

\section{RESUMEN}

\begin{abstract}
Se examinan los síntomas de estrés postraumático, ansiedad (rasgo-estado), afrontamiento a riesgos extremos y resiliencia psicológica en escolares que experimentaron el terremoto de México del 19 de septiembre de 2017. El presente es un estudio descriptivo prospectivo (longitudinal) con 130 niños de 11 a 15 años de edad, evaluados al mes (tiempo 1) y a los seis meses (tiempo 2) de ocurrido el evento. En la primera evaluación se alcanzaron puntajes más altos en las variables valoradas; mientras que en la segunda hubo un descenso, no obstante lo cual un tercio de la muestra aún manifestaba síntomas de estrés. En el tiempo 1, las niñas reportan puntuaciones más altas en síntomas de estrés y ansiedad (rasgo-estado) que los niños, diferencias que desaparecieron en el tiempo 2. Es necesario continuar llevando a cabo acciones que permitan la recuperación de los individuos a mediano y largo plazo, así como generar las condiciones necesarias para minimizar el impacto psicológico de tales desastres.
\end{abstract}

Palabras clave: Adaptación psicológica; Prevalencia; Catástrofes naturales; Vulnerabilidad; Riesgo.

\begin{abstract}
The present study examined symptoms of post-traumatic stress, anxiety (trait-state), coping with extreme risks, and psychological resilience in schoolchildren who experienced the earthquake occurred in Mexico on September 19, 2017. In this descriptive and prospective (longitudinal) study 130 boys and girls, between 11 and 15 years of age, were evaluated after a month (time 1) and after six months (time 2) since the earthquake. The first evaluation revealed higher scores in the assessed variables among girls and differences disappeared at the second measurement. One third of participants, however still remained showing symptoms of stress at time 2. It is necessary to continue carry out actions that allow the recovery of individuals in the middle and long terms, and promote conditions to minimize the psychological impact of such disasters.
\end{abstract}

Keywords: Psychological adaptation; Prevalence; Natural catastrophes; Vulnerability; Risk.

\footnotetext{
${ }^{1}$ Universidad Autónoma del Estado de México, Facultad de Ciencias de la Conducta. Filiberto Gómez s/n, Barrio de Tlacopa, 50010 Toluca, Edo. de México, México, tel: (722)272-00-76, ext. 157, correos electrónicos: nigalf@yahoo.com.mx y nigonzalezarratial@uaemex.mx. Artículo recibido el 14 de agosto de 2019 y aceptado el 27 de febrero de 2020.
} 


\section{INTRODUCCIÓN}

$\mathrm{D}$ esastres naturales como los terremotos son eventos súbitos e inesperados que pueden tener importantes consecuencias entre los habitantes de las zonas afectadas, siendo las más evidentes la pérdida de vidas humanas y las materiales y económicas. Sin embargo, también hay efectos psicológicos derivados de tales catástrofes que afectan, de manera directa o indirecta, la salud mental de las personas (Álvarez y Medina-Mora, 2018; Villamil, 2014), llevándolos a exhibir diversos síntomas conocidos como trastorno por estrés postraumático (TEPT por sus siglas en español).

El DSM-V (American Psychiatric Association [APA], 2014) indica que el TEPT se caracteriza por una serie de síntomas que se caracterizan por tener una duración superior a un mes y que incluyen "la reexperimentación de volver a vivir el evento traumático (p. ej. recuerdos y sueños angustiosos recurrentes, malestar psicológico intenso o prolongado), la evitación persistente de estímulos asociados al suceso y la alteración importante de la alerta y reactividad asociada al suceso (comportamiento irritable, hipervigilancia, problemas de concentración), los cuales causan malestar, dificultades para desenvolverse y relacionarse interpersonalmente" (APA, 2014).

Las investigaciones hechas en diversos países muestran una prevalencia de TEPT en población adulta expuesta a terremotos que varía de 10 a $74 \%$. Diez semanas después del ocurrido en México en 1985 se reportó una prevalencia de 32\%, mientras que, en el caso de los niños, las cifras oscilan en un rango de entre 18 y $70 \%$. En Lorca (España), en el año 2014, en una investigación sobre desastres naturales realizada por Derivois, Mérisier, Cénat y Castelot (2014) se observó una prevalencia al mes de ocurrido un terremoto de $55.4 \%$, y de $40.1 \%$ al año del mismo, donde se encontró que los niños fueron uno de los grupos más vulnerables (cf. También López y López, 2014; Tapia, Sepúlveda, Medina-Mora, Caraveo y De la Fuente, 1987).

Respecto al sexo, a nueve meses de vivenciar el terremoto de Chile en 2010 Caro y Cova (2012) indican que $10.2 \%$ de las mujeres manifestaron síntomas de TEPT, que en el caso de los hombres fue de $2.9 \%$. Tolin y Foa (2006) señalan que el TEPT es más frecuente en las mujeres y niñas que en los hombres y los niños, ya que posiblemente ellas tienden a experimentar eventos traumáticos más graves que ellos.

Del mismo modo, Derivois et al. (2014), en su estudio con niños y adolescentes después del sismo de Haití (2010), hallaron indicadores de TEPT más altos en las mujeres, y explican que el soporte social y la resiliencia pueden reducir los síntomas. Así, el sexo, la edad y la repetición del suceso traumático son factores asociados con la presencia de TEPT (Tolin y Foa, 2006).

De acuerdo con Kaya (2013), en la infancia la ansiedad es otra de las reacciones más frecuentes ante los desastres. La ansiedad es un estado de agitación e inquietud desagradable que se define por una anticipación del peligro, y que se concibe como rasgo, es decir, una propensión ansiosa relativamente estable que caracteriza a los individuos con tendencia a percibir las situaciones como amenazadoras, o bien como estado emocional transitorio y variable en su intensidad y duración que es vivenciada por el individuo como patológico en un momento particular (Sierra, Ortega y Zubeidat, 2003). En 2010, en el terremoto de Chile, el número de consultas por los trastornos de ansiedad aumentó levemente en las regiones afectadas durante los meses posteriores al suceso (Bambarén, 2011).

No obstante, también se ha evidenciado que muchas personas, a pesar de estar expuestas a eventos traumáticos, no necesariamente desarrollan tales trastornos (Carr, Lewin, Webster y Kenardy, 1997), y que incluso pueden aprender de dichas experiencias, desenvolviéndose psicológicamente fuertes y socialmente exitosas. A estos individuos se les denomina resilientes y se caracterizan por una serie de atributos, como buscar activamente soluciones y mostrar capacidad de afrontamiento, la cual alude a dar las respuestas conductuales necesarias para hacer frente a las demandas del medio.

Según López y Marván (2004), las estrategias de afrontamiento en estos casos son dos: la activa, que se refiere a comportamientos de acción directa sobre el problema, y la pasiva, que alude a comportamiento de rechazo, de negación del evento, de retraimiento y de aceptación pasiva. Estos mismos autores señalan que los adultos que experimentaron el terremoto de México de 1985 
enfrentaron la situación mediante la aceptación de las pérdidas, y en el caso del terremoto de Chile de 2010 quienes afrontaron los desastres con un estilo pasivo o con estrategias de evitación mostraron una resiliencia parcial (Villagrán, Reyes y Wlodarczyk, 2010); además, se ha observado que los adolescentes que muestran estrategias de afrontamiento pasivas expresan una capacidad menor para resolver problemas (Salotti, 2006).

México es considerado como un país vulnerable a diversos fenómenos naturales, en donde son los sismos los que más defunciones han causado. Lo anterior implica importantes pérdidas económicas, un aumento imprevisto del gasto en el sector salud y elevados costos en la reparación de los daños, lo que afecta drásticamente a las poblaciones vulnerables (Abeldaño y González, 2018), por lo que es indispensable una adecuada preparación ante tales desastres.

El 19 de septiembre de 2017, en México ocurrió un terremoto de magnitud 7.1 en escala de Richter, localizado entre los estados de Puebla y Morelos, con epicentro localizado a 12 kilómetros al sureste de Axichiapan, Mor., y a 120 de la Ciudad de México, cuyo sitio de aceleración máxima registrada fue en Tlamacas, Estado de México, registrándose además múltiples réplicas, según lo informado por el Servicio Sismológico Nacional (2017). Específicamente en el Estado de México, se informó un saldo de 13 personas fallecidas, 800 viviendas destruidas, 1,500 de estas con diversos daños y 600 escuelas afectadas; entre los municipios que sufrieron más daños se encuentra precisamente Malinalco, lugar donde se llevó a cabo el presente estudio.

Estudios internacionales han mostrado que ha habido un importante aumento de daños ocasionados por los desastres naturales, lo que se debe a múltiples factores, entre los que se cuentan el crecimiento de la población en áreas costeras y de riesgo y variaciones climáticas extremas, tales como inundaciones y sequías. Lo anterior ha llevado a que a la fecha exista una vasta literatura sobre tales fenómenos (Pierro, 2018). Sin embargo, en México aún es escasa la investigación al respecto $\mathrm{y}$, en consecuencia, la publicación de hallazgos; por consiguiente, no se han publicado los necesarios trabajos sobre el impacto y las conse- cuencias psicológicas en niños y adolescentes tras el mencionado terremoto en los que se incluyan indicadores de estrés postraumático, ansiedad, afrontamiento ante los riesgos extremos y resiliencia, sobre todo porque se requiere analizar la capacidad de los individuos para sobreponerse y adaptarse después de una experiencia traumática. En virtud de que también la investigación ha mostrado que los efectos traumatizantes pueden perdurar un largo tiempo (Chemtob, Nakashima y Carlson, 2002, Domínguez, 2017), se requiere profundizar en estas variables para analizar si persisten o disminuyen los síntomas, lo que permitiría estimar la tasa de cambio en función del tiempo (Arnau y Ono, 2008).

La mayoría de los estudios sobre TEPT evalúan a los niños más de un mes después del evento traumático, lo que, de acuerdo con Roemer, Litz, Orsillo, Ehlich y Friedman (1998), reduce su confiabilidad, por lo que se debería realizar un seguimiento de las trayectorias de las respuestas, a lo que Tolin y Foa (2006) recomiendan efectuar estudios longitudinales a partir del evento y determinar los factores que hacen que algunos niños desarrollen trastornos postraumáticos más persistentes que otros (La Greca, Silverman, Vernberg y Roberts, 2002).

Así, los objetivos de la presente investigación fueron los de describir la sintomatología de estrés postraumático, ansiedad, estilos de afrontamiento frente a riesgos extremos y resiliencia psicológica en escolares que vivenciaron el terremoto de México del 19 de septiembre de 2017; comparar las observaciones de un mismo individuo en dos momentos distintos de tiempo -específicamente al mes y seis meses de ocurrido el terremoto-, para determinar si variaban significativamente en ambos momentos, y examinar si había diferencias significativas entre niños y niñas respecto a cada variable en esos dos momentos.

La importancia de esta investigación radica en que estos datos pueden ser útiles para la toma de decisiones que permitan atender la salud mental en niños en casos de desastres en el corto y el largo plazo, así como mostrar información científica para generar grupos de trabajo a fin de fomentar la resiliencia en las comunidades afectadas por dichos sucesos. 


\section{MÉTODO}

\section{Participantes}

Este estudio, de tipo descriptivo prospectivo (longitudinal), implica dos o más momentos temporales en el que un mismo individuo es evaluado (Ato, López y Benavente, 2013). El primer tiempo, T1, ocurre al mes de ocurrido el evento, y el segundo, T2, a los seis meses. Los participantes en el T1 fueron 178 niños de ambos sexos, quienes participaron asimismo en un estudio transversal previo (González-Arratia, Torres, Aguilar, González-Arratia, González y Ruiz, 2019), de los cuales 85 fueron hombres y 93 mujeres. Debido a que es frecuente la pérdida en el número de participantes en este tipo de trabajos, en el T2 solo completó la segunda aplicación $73 \%$ de los 178 evaluados inicialmente, por lo que fue necesario hacer análisis subsecuentes de los datos (APA, 2014). Las variaciones en la tasa de respuesta entre el T1 y el T2 se debieron a diversas razones, entre las que destacan que la mayoría no se encontraba en la institución al momento de la segunda aplicación y, en menor medida, que algunos no aceptaron participar de nuevo en la investigación, lo que implicó que 48 casos quedaran incompletos en el T2, motivo por el cual se decidió excluirlos del estudio. De este modo, la muestra del presente estudio quedó constituida por un total de 130 participantes, de los cuales 64 fueron varones $(49.2 \%)$ y 66 niñas $(50.8 \%)$, con un rango de edad de 11 a 15 años $(\mathrm{M}=13.03$, D.E. $=.95)$. Todos eran alumnos de nivel básico de una escuela pública ubicada en San Simón, municipio de Malinalco, que es una de las zonas del Estado de México que resultó con importantes daños después del mencionado sismo.

\section{Instrumentos}

\section{Escala Infantil de Síntomas del Trastorno}

de Estrés Postraumático (CPSS)

(Foa, Johnson, Feeny y Treadwell, 2001).

Se aplicó la versión validada en niños por Rincón et al. (2014). Contiene 17 síntomas que conforman el diagnóstico, y tres subescalas: Reexperimentación (cinco ítems), Evitación (siete) y Activación (cinco), que se califican en una escala de cinco puntos, que va de "nunca" a "nueve veces y más", y que tiene una fiabilidad de 0.916. De acuerdo con el punto de corte, se considera como alto TEPT un puntaje igual o superior a 24 y bajo con menos de 24 puntos.

Ansiedad Estado-Rasgo en Niños (STAIC) (Spielberger, Goursch y Lushene, 1982).

Para evaluar la ansiedad se aplicó este cuestionario de autoevaluación. La escala A-E consta de veinte ítems y tres opciones de respuesta ("nada", "algo" y "mucho"). La escala A-R, a su vez, contiene veinte ítems, y las respuestas expresan la frecuencia ("casi nunca", "a veces" y "a menudo"). La puntación mínima en cada escala es de 20 y la máxima de 60 puntos. Sus autores reportan fiabilidades de 0.91 y de 0.87 para la ansiedad-estado y la ansiedad-rasgo, respectivamente.

Escala de Resiliencia (González-Arratia, 2016).

Esta escala consta de treintaidós ítems y se califica en un formato de respuesta tipo Likert de cinco puntos, donde el valor 1 indica "nunca" y el 5 "siempre". Con una varianza total explicada de $40.33 \%$ y una consistencia interna medida con el coeficiente alfa de Cronbach de 0.91, contiene tres dimensiones: factores protectores internos (PFI), factores protectores externos (FPE) y factor de empatía (FE).

Escala de Afrontamiento frente a Riesgos Extremos (López y Marván, 2004).

Este instrumento consta de veintiséis ítems y dos factores: factor 1, afrontamiento activo, con catorce ítems ( $\alpha=0.79$ ), y factor 2 , afrontamiento pasivo, con doce ítems $(\alpha=0.76$.) con $31.65 \%$ de la varianza total.

Además, se elaboró una ficha de datos sociodemográficos, que incluye sexo, edad y escolaridad. Todos los instrumentos fueron identificados con fines de seguimiento.

\section{Procedimiento}

Primeramente, se solicitó el consentimiento informado de los padres, el consentimiento de los participantes y la autorización de la institución, a quienes se explicó el objetivo de la investigación. Una vez obtenido el permiso, se programó la aplicación, que se realizó en las respectivas aulas y horarios académicos de manera colectiva. La estrategia utilizada fue la misma en ambas aplica- 
ciones. Después, se procedió a realizar la evaluación, la cual estuvo a cargo de los mismos autores de este estudio.

Al momento de las aplicaciones se solicitó a los participantes que tuvieran presente el terremoto como evento y que posteriormente describieran su sintomatología (al responder a las escalas), con la intención de que fuera la misma situación y no un evento en particular. Se procedió a recolectar e identificar los instrumentos de ambas aplicaciones, y todas las respuestas fueron registradas en el T1 y T2, teniendo en cuenta que la información de ambas aplicaciones pertenecen al mismo participante.

El tiempo de las aplicaciones fue de aproximadamente 60 minutos para cada una. Cabe señalar que todos participaron de manera voluntaria y que las aplicaciones del T1 se llevaron a cabo en el mes de octubre y las de $\mathrm{T} 2$ en el mes de abril del año siguiente. Esta investigación se realizó bajo los estándares éticos que indica la APA (2014) y de manera acorde con lo que se establece en la Declaración de Helsinki. Los datos se analizaron con el programa SPSS, versión 20, considerándose una significancia de 0.05 en cada prueba.

\section{RESULTADOS}

Con base en el puntaje total de la escala de TEPT, y según el criterio de Rincón et al. (2014), todos los participantes fueron clasificados con y sin síntomas de TEPT. En el caso del T1 se encontró una prevalencia del $50.77 \%(\mathrm{n}=66)$ de la muestra con algún tipo de síntoma de estrés, mientras que en el T2 fue del $33.85 \%(\mathrm{n}=44)$, de los cuales 23 fueron niños y 21 niñas.

Se muestran primeramente los datos obtenidos de la prueba $t$ de Student para muestras relacionadas, observándose diferencias estadísticamente significativas en los síntomas de reexperimentación y activación y en el puntaje total, así como en afrontamiento pasivo y en las tres dimensiones de la Escala de Resiliencia. Hecho lo anterior, se examinó la magnitud del efecto de las diferencias a través del cálculo de la $d$ de Cohen, encontrándose un efecto pequeño en afrontamiento de tipo pasivo y en reexperimentación, mientras que en la variable resiliencia la magnitud del efecto fue mayor (Tabla 1).

Tabla 1. Afrontamiento, ansiedad, estrés postraumático y resiliencia. Diferencias entre T1 y T2.

\begin{tabular}{|c|c|c|c|c|c|c|c|c|c|c|}
\hline & \multirow{2}{*}{\multicolumn{3}{|c|}{$\begin{array}{c}\mathrm{T} 1 \\
\text { A un mes del sismo } \\
(\mathrm{N}=130)\end{array}$}} & \multirow{2}{*}{\multicolumn{3}{|c|}{$\begin{array}{c}\mathrm{T} 2 \\
\text { A seis meses del sismo } \\
(\mathrm{N}=130)\end{array}$}} & \multirow{3}{*}{$t$} & \multirow{3}{*}{$p$} & \multirow{3}{*}{$\begin{array}{c}d \text { de } \\
\text { Cohen }\end{array}$} \\
\hline & & & & & & & & & & \\
\hline Variables & Dimensiones & $\begin{array}{l}\text { Min- } \\
\text { máx. }\end{array}$ & M & DE & $\begin{array}{l}\text { Min- } \\
\text { máx. }\end{array}$ & $\mathbf{M}$ & DE & & & \\
\hline \multirow{2}{*}{ Afrontamiento } & Activo & $18-64$ & 40.28 & 8.8 & $16-66$ & 40.66 & 9.0 & -.35 & .725 & .060 \\
\hline & Pasivo & $23-47$ & 33.19 & 5.3 & $16-50$ & 31.70 & 6.1 & 2.09 & $.038 *$ & 0.26 \\
\hline \multirow{2}{*}{ Ansiedad } & Rasgo & $21-55$ & 35.88 & 6.5 & $23-73$ & 34.57 & 7.4 & 1.49 & .138 & 0.18 \\
\hline & Estado & $20-48$ & 32.07 & 6.4 & $21-50$ & 30.77 & 6.4 & 1.53 & .127 & 0.20 \\
\hline \multirow{4}{*}{$\begin{array}{l}\text { Síntomas de estrés } \\
\text { postraumático }\end{array}$} & Reexperimentación & $0-19$ & 8.13 & 4.8 & $0-20$ & 6.65 & .3 & 2.20 & $.029 * *$ & 0.29 \\
\hline & Evitación & $0-24$ & 9.14 & 5.8 & $0-24$ & 7.99 & 6.2 & 1.45 & .147 & 0.19 \\
\hline & Activación & $0-20$ & 7.37 & 5.2 & $0-20$ & 5.46 & 4.5 & 3.12 & $.002 * *$ & 0.39 \\
\hline & Puntaje total & $0-58$ & 24.75 & 14.0 & $0-61$ & 20.02 & 14.4 & 2.41 & $.017 * *$ & 0.33 \\
\hline \multirow{3}{*}{ Resiliencia } & FPI & $2-5$ & 3.76 & .58 & $1-3$ & 2.05 & .54 & 26.10 & $.000 * *$ & 3.05 \\
\hline & FPE & $2-4$ & 3.94 & .59 & $1-4$ & 1.98 & .65 & 24.53 & $.000 * *$ & 3.15 \\
\hline & $\mathrm{FE}$ & $1-5$ & 3.66 & .73 & $1-4$ & 2.19 & .74 & 15.64 & $.000 * *$ & 1.99 \\
\hline \multicolumn{2}{|l|}{ Puntaje total } & $69-156$ & 121.83 & 17.9 & $34-119$ & 65.95 & 17.4 & 25.81 & $.000 * *$ & 3.16 \\
\hline
\end{tabular}

Con la finalidad de examinar las diferencias en función de la variable sociodemográfica de sexo, se realizó un análisis de $t$ de Student de muestras independientes. En el caso de T1 se observan diferencias significativas en seis de las doce variables evaluadas. Las variables significativas con puntajes promedios más altos fueron, en el caso de las niñas, ansiedad- rasgo, ansiedad-estado y TEPT; en comparación con los niños y en la magnitud del efecto, tales diferencias resultaron ser signifi- 
cativas. Respecto al T2, ninguna de las variables evaluadas alcanzó a ser significativa, por lo que no se reportan diferencias respecto al sexo ni a la edad (Tabla 2).

Tabla 2. Afrontamiento, Ansiedad, Estrés Postraumático y Resiliencia. Diferencias según sexo a 1 mes y 6 meses del sismo.

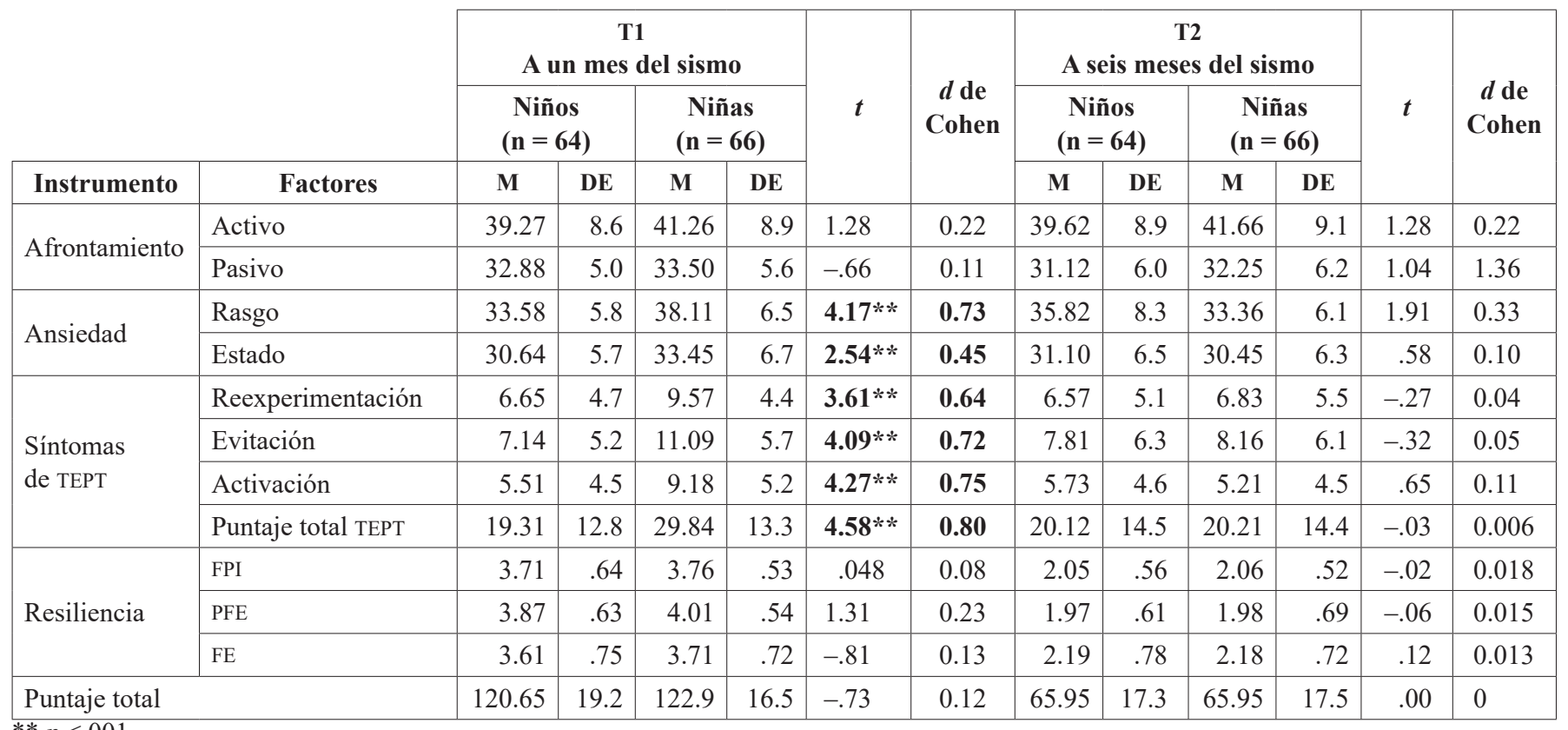

\section{DISCUSIÓN}

Los resultados del presente estudio revelan la presencia de síntomas de TEPT en el primer momento en que fueron evaluados los participantes, mismos que disminuyeron en la segunda aplicación, lo que coincide con los resultados de Kronenberg et al. (2010). Respecto a la primera evaluación, es importante considerar que la presencia de un nivel más alto de TEPT puede deberse a las reacciones iniciales normales ante la exposición directa o indirecta al suceso, pues es posible que surjan pesadillas, recuerdos, síntomas físicos, e insomnio, que son los síntomas propios del TEPT (APA, 2014; Caro y Cova, 2012).

En la segunda medición los TEPT disminuyeron; en particular, la dimensión Activación fue el criterio menos prevalente, lo cual está de acuerdo con lo hallado por López y López (2011); además, se tiene que tomar en cuenta que la prevalencia evaluada en este estudio fue significativamente menor que lo reportado en otras investigaciones (p. ej. Caro et al., 2012; Derivois et al., 2014; López y López, 2011), lo que puede ser un indicador de que un mayor porcentaje de participantes de este trabajo tienen al parecer un mejor funcionamiento.

En el caso de la reexperimentación, las diferencias obtenidas entre el T1 y el T2 fueron significativas y que el tamaño del efecto de dichas diferencias se considera moderado, lo que podría explicarse en el sentido de que la frecuencia de esta sintomatología disminuyó a través del tiempo. Tales hallazgos concuerdan con los de otros estudios, como el de Piña, Torres, Prawd y Pérez (1987), quienes investigaron los TEPT en estudiantes de medicina a las dos semanas y ocho meses posteriores al terremoto de México. En su trabajo reportaron que, a pesar de que en la mayoría de los encuestados la sintomatología disminuyó a los ocho meses del evento, $10 \%$ de ellos aún padecía temor o angustia.

Merece la pena señalar que en el presente artículo los síntomas aún persistían a los seis meses posteriores del sismo en algunos casos, lo que también se ha evidenciado en estudios de seguimiento, que explican que puede haber condiciones crónicas relacionadas con el estrés después de la exposición a esos fenómenos (Chemtob et al., 2002; Goenjian et al., 2000, La Greca et al., 
2002). En esos casos pueden persistir en el largo plazo los efectos negativos en el pensamiento y el sentimiento de los niños traumatizados (Celebi, Metindongan y Wise, 2010), así como importantes consecuencias psicológicas que pueden ser de larga duración. Se ha reportado que muchos sobrevivientes de experiencias traumáticas sufren no solamente los síntomas relacionados, sino también depresión y abuso de sustancias (Cohen, Mannarino, Berliner y Deblinger, 2000; Palomares y Campos, 2018). Incluso las reacciones al trauma puedan manifestarse como problemas de conducta, o bien de salud, por lo que es indispensable analizar estos aspectos aun en ausencia de TEPT.

De ahí que sería pertinente continuar observando tales reacciones debido a que es posible que existan casos en los que, transcurrido un tiempo prolongado tras el suceso, aparezca algún tipo de sintomatología, por lo que hay la necesidad de vigilar a las personas expuestas en el caso de los desastres naturales, continuando su asesoramiento para así evitar problemas subsecuentes de salud mental (Maclean et al., 2016).

En cuanto a la ansiedad, si bien no se hallan diferencias estadísticas, en la mayoría de los participantes hay al parecer una recuperación (Chou et al., 2004), por lo que los TEPT disminuyen con el tiempo, sobre todo en personas que experimentan esos desastres naturales.

Estos hallazgos coinciden asimismo con los de Bonanno (2004), en el sentido de que por lo general el TEPT gradualmente regresa, en un periodo de varios meses, a los niveles previos al evento. Además, se ha reportado que la mayoría de los individuos no exhiben perfiles de síntomas crónicos, ya que en general muestran un funcionamiento saludable; dicho autor refiere que $85 \%$ de los individuos siguen un proceso de recuperación natural y no desarrollan ningún tipo de trastorno, por lo que es relevante prestar atención al paso del tiempo entre el evento y la medición que se haga.

En el caso de la resiliencia, el tamaño del efecto de las diferencias fue elevado. Los datos mostraron una disminución en la segunda medición, lo que puede ser un proceso de recuperación natural, tal como lo señalan Bisson, Jenkins, Alexander y Bannister (1997). Al respecto, según Bonanno (2004), los individuos resilientes pueden experimentar perturbaciones transitorias en el funcionamiento, para después mostrar un funcionamiento estable; de hecho, la mayoría se recupera de forma natural y en un tiempo breve recobra su nivel normal de funcionamiento (cf. Goenjian et al., 2000). Del mismo modo, estas diferencias pueden deberse a diversos factores, como el apoyo social y familiar con el que cuentan los menores, lo cual será necesario incluir en la siguiente medición y constatar si hay un patrón de rehabilitación en términos de resiliencia, de disfunción crónica o de retraso en la recuperación del trauma.

Se encontraron diferencias significativas en el afrontamiento a los desastres en función del tiempo: al mes de ocurrido el evento, los niños utilizan con mayor frecuencia un estilo de afrontamiento pasivo. Una de las posibles explicaciones de lo anterior es que el suceso era relativamente reciente, y que dicho estilo resultó ser un mecanismo de adaptación adecuado en ese momento. Además, es posible considerar que los participantes de este trabajo tendían a responder a los eventos traumáticos de manera fatalista, lo que es un patrón común en el contexto latinoamericano (Pole, Gone y Kulkami, 2008). Sin embargo, este resultado lleva a considerar la necesidad de realizar otros estudios sobre la eficacia del uso de un determinado estilo de afrontamiento tras un desastre, pues si bien en un momento puede ser útil una forma de enfrentar la situación, no necesariamente lo es en otro.

Respecto al sexo, no se presentaron diferencias entre los niños y las niñas, lo que fue consistente también a los seis meses. Desde una perspectiva desarrollista, puede ser que surjan diferencias conforme los niños crecen (Zwirs et al., 2007), por lo que se requiere continuar analizando esta importante variable según el modo en que se interpreta la situación, así como su asociación con la edad y el sexo.

Los resultados de este estudio sugieren continuar con el seguimiento, por lo que se recomienda que los servicios de salud mental deberían extenderse durante un tiempo considerable, más allá del periodo de crisis en los niños que aún continúan con un TEPT; en efecto, los participantes reportaron que no habían recibido hasta ese momento ningún tipo de intervención psicológica, lo que es un reto del sector salud para el apoyo a la población. Así, dada la relevancia de atender los síntomas psicológicos como resultado de vi- 
venciar este tipo de desastres, se requiere poner en práctica acciones decisivas a través de dichas intervenciones. La literatura al respecto recomienda frecuentemente la terapia cognitivo-conductual (Palomares y Campos, 2018), y aunque se ha probado su eficacia, también sería recomendable diseñar programas para el desarrollo de la resiliencia ante los riesgos naturales. Del mismo modo, es indispensable contar con servicios de salud que cuenten con los mecanismos idóneos para responder en situaciones críticas y mediante planes de acción consecuentes con las necesidades de atención (Álvarez y Medina-Mora, 2018).

Una importante limitación de esta investigación es que únicamente se consideró la aplicación de instrumentos del tipo de autorreportes, por lo que se sugiere la inclusión de entrevistas clínicas a fin de verificar la presencia de los síntomas de TEPT. De ahí que el próximo paso será explorar la manera en que se recuperan los individuos tras un trauma, para después analizar las habilidades conductuales en situaciones de incertidumbre, y así indagar sobre cómo se encontraban los niños antes del suceso, y de esta manera tener una explicación más amplia sobre la resiliencia psicológica en la infancia, ya que, según los resultados, los participantes pudieron experimentar un cambio positivo; cabe señalar a pesar de la experiencia traumática, la mayoría no manifestaba síntomas psicopatológicos.

Es necesario reconocer ciertos inconvenientes metodológicos en esta investigación, como el intervalo de tiempo transcurrido entre las mediciones, que fue relativamente corto, así como el hecho de que solo fueron dos puntos en el tiempo, lo que no permitió considerar el efecto de la edad sobre la prevalencia de TEPT. Sin embargo; las variaciones entre el $\mathrm{T} 1$ y $\mathrm{T} 2$ muestran que hubo una disminución de los síntomas, lo cual es posible atribuir al mero paso del tiempo, lo que implica la necesidad de considerar una siguiente medición para contar con una mayor evidencia empírica que permita explicar el fenómeno bajo estudio.

Finalmente, conviene señalar que las respuestas de los participantes ante los desastres naturales pueden estar asociadas no solo al TEPT, sino a otros trastornos, por lo que la ocurrencia de otro evento puede desencadenar el recuerdo perturbador y afectar el equilibrio emocional, por lo que es indispensable tomar en cuenta no solo el tipo de suceso, sino también la gravedad, la intensidad y la proximidad respecto al primero, pero también el apoyo percibido y los factores subjetivos debidos al trauma, los cuales podrán determinar la presencia de TEPT en la infancia.

\section{REFERENCIAS}

Abeldaño, R.A. y González, A.M. (2018). Desastres en México de 1900 a 2016: patrones de ocurrencia, población afectada y daños económicos. Rev Panam Salud Publica, 42e55. https://Doi.org/10.2633/RPSP.2018.55.

Álvarez I., D. y Medina-Mora, M.E. (2018). Impacto de los sismos de septiembre de 2017 en la salud mental de la población y acciones recomendadas. Salud Pública Mex., 60(Supl. 1). 52-58.

American Psychiatric Association (2014). Diagnostic and Statistical Manual of Mental Disorders (5 ${ }^{\mathrm{a}}$ ed.). Washington, D.C.: Autor.

Arnau, J. y Ono, R. (2008). Estudios longitudinales. Modelos de diseño y análisis. Escritos de Psicología, 2(1), 32-41.

Ato, M., López, J.J. y Benavente, A. (2013). Un sistema de clasificación de los diseños de investigación en psicología. Anales de Psicología, 29(3), 1038-1059.

Bambarén, C. (2011). Salud mental en desastres naturales. Revista Psicológica Herediana, 6(1-2), 20-25.

Bisson, J., Jenkins, P.L., Alexander, J. y Bannister, C. (1997). Randomised controlled trial psychological debriefing for victims of burn trauma 1997. British Journal of Psychiatry, 171, 78-81. Doi:10.1192/bjp.171.1.78.

Bonanno, G.A. (2004). Loss, trauma and human resilience: Have we underestimated the human capacity to thrive after extremely aversive events? American Psychologist, 59(1), 20-28.

Caro M., P.P. y Cova S., F.M. (2012). Relación entre estrés postraumático, afrontamiento y rumiación en estudiantes universitarios chilenos. Ciencia y Enfermería, 18(3), 121-130. Doi: 10.4067/S0717-95532012000300012.

Carr, V.J., Lewin, T.J., Webster, R.A. y Kenardy, J. (1997). A synthesis of the findings from the Quake Impact Study: A two-year investigation of the psychosocial sequelae of the 1989 Newcastle earthquake. International Journal of Social Psychiatry and Psychiatric Epidemiology, 32, 123-136. 
Celebi, E., Metindogan, A. y Wise, A. (2010). The effects of the 1999 Turkishh earthquake on young children: Analyzing traumatized children's completion of short stories. Child Development, 81(4), 1161-1175.

Chemtob, C.M., Nakashima, J. y Carlson, J.C. (2002). Brief treatment for elementary school children with disaster-related posttraumatic stress disorder: A field study. Journal of Clinical Psychology, 58, 99-112.

Chou, F.H., Chou, P., Su, T.T., Ou-Yang, W.C., Chien, I.C., Lu, M.K. y Huang, M.W. (2004). Quality of life and related risk factor in a Taiwanesse village population at 21 months after the earthquake. Australian \& New Zealand Journal of Psychiatry, 58(1), 358-364.

Cohen, J.A., Mannarino, A.P., Berliner, L. y Deblinger, E. (2000). Trauma-focused cognitive behavioral therapy for children and adolescents: An empirical update. Journal of Interpersonal Violence, 15(11), 1202-1223. Doi: 10.1177/08862600001501007.

Derivois, D., Mérisier, G.G, Cénat, J.M. y Castelot, V. (2014). Symptoms of posttraumatic stress disorder and social support among children and adolescents after the 2010 Haitian earthhquake. Journal of Loss and Trauma, 19, 202-212. Doi: 20.1080/15325024.2013.789759.

Domínguez T., B. (2017). Estrés postraumático, entre los efectos del sismo. Gaceta Universitaria, 5(106). Recuperado de http:// gaceta.unam.mx/estres-postraumatico-entre-los-efectos-del-sismo/.

Foa, E., Johnson, K., Feeny, N. y Treadwell, K. (2001). The Child PTSD Symptom Scale: A preliminary examination of its psychometric properties. Journal of Clinical Child Psychology, 30, 376-384. Doi: 10.1207/s15374424JCCP3003_9.

Goenjian, A.K., Steinberg, A.M., Najarian, L.M., Fairbanks, L.A., Tashjian, M. y Pynoos, R.S. (2000). Prospective study of posttraumatic stress, anxiety, and depressive reactions after earthquake and political violence. American Journal of Psychiatry, 157(6), 911-916.

González-Arratia L.F., N.I. (2016). Resiliencia y personalidad en niños y adolescentes. Cómo desarrollarse en tiempos de crisis. México: Eón-Universidad Autónoma del Estado de México.

González-Arratia L.F., N.I., Torres M., M.A., Aguilar M., O., González-Arratia, V., González E., S. y Ruiz M., A.O. (2019). Afrontamiento frente a riesgos extremos y síntomas de estrés postraumático en mexicanos después que vivenciaron el sismo del 2017. Ciencia ErgoSum, 6(3), https://Doi.org/10.30878/ces.v26n3a3.

Kaya, E. (2013). Effects of disaster on children health. TAF Preventive Medicine Bulletin, 12(4), 455-464.

Kronenberg, M.E., Hansel, T.C., Brennam, A.M., Osofsky, H.J., Osofsky, J.D. y Lawrason, B. (2010). Children of Katrina: lessons learned about symptoms and recovery patterns. Child Development, 81(4), 1241-1259.

La Greca, A.M., Silverman, W.R., Vernberg, E.M. y Roberts, M.C. (2002). Helping children cope with disasters and terrorism. Washigton, D.C.: American Psyhological Association.

López G., J. y López S., C. (2014). Trastorno de estrés postraumático en escolares tras el terremoto de Lorca (España) en 2011. Gaceta Sanitaria, 28(3), 230-233.

López V., E. y Marván, M.L. (2004). Validación de una escala de afrontamiento frente a riesgos extremos. Salud Pública de México, 46, 216-221.

Maclean, J.C., Popovici, I. y French, M.T. (2016). Are natural disasters in early childhood associated with mental health and substance use disorders as an adult? Social Science \& Medicine, 151, 78-91.

Palomares C., E. y Campos C., P.E. (2018). Impacto de los terremotos en la salud mental. Ciencia, 60(3), 48-55.

Pierro, B. (2018). La ciencia de los desastres. Pesquisa FAPESP, Edición 264, febrero. Recuperado de https://revistapesquisa. fapesp.br/es/2019/07/29/la-ciencia-de-los-desastres/.

Piña B., B., Torres C., J.L., Prawda W., M. y Pérez R., G. (1987). Efectos psicológicos causados por los sismos de 1985 en estudiantes de medicina. Trabajo presentado en el Tercer Congreso Mexicano de Epidemiología, Tuxtla Gutiérrez, Chis., 6 de agosto de 1987.

Pole, N., Gone, J.P. y Kulkami, M. (2008). Posttraumatic stress disorder among ethnoracial minorities in the United States. Clinical Psychology: Science and Practice, 15, 35-61.

Rincón, P., Gysling, M., Jiménez, C., Lloyd, S., Navarro, M.F., Retamal, L., Cova, F., Valdivia, M., Paz, P-S.C., Melipillán, R. y Haquin, C. (2014). Propiedades psicométricas de la Escala de Síntomas de TEPT para niños (CPSS) en población chilena afectada por el terremoto y tsunami del 27-F de 2010. Terapia Psicológica, 32(1), 57-64.

Roemer, L., Litz, B.T., Orsilo, S.M., Ehlich, P.J. y Friedman, M.J. (1998). Increases in retrospective accounts of war-zone exposure over time, the role of PTSD symptom severity. Journal of Trauma \& Stress, 11(3), 597-605. Doi: 10.1023/A:1024469116047.

Salotti, P. (2006). Estudio sobre estrategias de afrontamiento y bienestar psicológico en una muestra de adolescentes de Buenos Aires. Tesis para optar la licenciatura en Psicología. Buenos Aires: Universidad de Belgrano. Recuperado de http://www. ub.edu.ar/investigaciones/tesinas/207_salotti.pdf.

Servicio Sismológico Nacional (2017). Reporte Especial del Grupo de Trabajo del Servicio Sismológico Nacional, UNAM. Sismo del día 19 de septiembre de 2017, Puebla-Morelos (M 7.1). México: SSN. Recuperado de http://www.ssn.unam.mx/sismicidad/reportes-especiales/2017/sSNMX_rep_esp_20170919_Puebla-Morelos_M71.pdf.

Sierra, J.C., Ortega, V. y Zubeidat, I. (2003). Ansiedad, angustia y estrés: tres conceptos a diferenciar. Mal-Estar e Subjetividade, $3(1), 10-59$. 
Spielberger, C.D., Goursch, R.L. y Lushene, R.E. (1982). Cuestionario de Ansiedad Estado-Rasgo, STAI. Madrid: TEA Ediciones.

Tapia, R., Sepúlveda, J., Medina-Mora, M.E., Caraveo, J. y De la Fuente, J.R. (1987). Prevalencia del síndrome de estrés postraumático en la población sobreviviente a un desastre natural. Salud Pública de México, 29(5), 406-411.

Tolin, D.F. y Foa, B.E. (2006). Sex differences in trauma and posttraumatic stress disorder: a quantitative review of 25 years of research. Psychological Bulletin, 32(6), 959-992.

Villagrán, L., Reyes, C. y Wlodarczyk, A. (2014). Afrontamiento comunal, crecimiento postraumático colectivo y bienestar social en el contexto del terremoto del 27 de febrero de 2010 en Chile. Terapia Psicológica, 32(3), 243-254.

Villamil, V. (2014). La salud mental ante los desastres. Salud Mental, 37, 363-364.

Zwirs, B., Burger, H., Schilpen, T., Wiznitzer, M., Fedder, H. y Buotelaar, J. (2007). Prevalence of psychiatric disorders among children of different ethic origin. Journal of Abnormal Child Psychology, 35, 556-566. Doi: 10.1007/s10802-007-9112-9. 